\title{
Undergraduates' Barriers to Online Learning During the Pandemic in Greece
}

\author{
Marinos Anastasakis ${ }^{1,2}$ (D) $\cdot$ Georgios Triantafyllou ${ }^{3} \cdot$ Konstantinos Petridis $^{2,4}$
}

Accepted: 22 November 2021

(C) The Author(s), under exclusive licence to Springer Nature B.V. 2021

\begin{abstract}
In this paper we explore the difficulties undergraduates from Greek Higher Educational Institutions faced during the transition to online learning due to the COVID-19 pandemic. By analyzing qualitative data from an online survey $(N=2093)$, we were able to identify the barriers undergraduates encountered during this transition and make inferences about the quality of the way online learning was implemented. The most frequently reported barriers include problems that undergraduates perceived as being caused by their lecturers, internet connection issues during an online lecture and the perceived limited social interactions that synchronous environments can afford. The paper argues that the way online learning was implemented by Greek Higher Educational Institutions did not result a meaningful learning experience for undergraduates. Our results are relevant to universities that are either forced in implementing emergency online protocols or wish to support their undergraduates' learning experience.
\end{abstract}

Keywords Barriers · Online learning · Undergraduates · Transactional distance · COVID19

\section{Introduction}

Over the course of the last 20 years, the popularity of online learning has steadily increased and many universities consider now this form of instruction as an integral part of the undergraduate experience (Dumford \& Miller, 2018) but more importantly critical for the future of higher education (Kebritchi et al., 2017). Although not all universities have chosen to deliver course content in a fully online manner, the declaration of COVID-19 as a pandemic in March 2020 changed everything: the restrictions posed during the first lockdown

Marinos Anastasakis

m.anastasakis@iacm.forth.gr

1 Institute of Applied and Computational Mathematics, Foundation for Research and Technology Hellas (FORTH), Heraklion, Greece

2 Department of Electronic Engineering, Hellenic Mediterranean University, Chania, Greece

3 Department of Tourism and Management, Hellenic Mediterranean University, Heraklion, Greece

4 ATHENA European University, Heraklion, Greece 
period led universities across the globe to respond with "emergency eLearning" protocols, which marked the rapid transition from face-to-face to online learning (Murphy, 2020). During that period, undergraduates faced a number of challenges, but arguably, the greater challenge they faced as learners was the rapid transition to a different mode of learning: almost overnight, they had to adapt and become familiar with the mechanics of distance education (Naidu, 2020). Given the urgent character of this transition, it is reasonable to assume that undergraduates from countries where distance education was not a well-established didactical approach faced more challenges than their peers from other regions of the world where this modality of learning had a longer tradition. One such example is Greece.

The interest in online teaching and learning in Greece has grown since the 2000s mainly as a national priority to increase the quantity, quality and effectiveness of investment in human capital in order to upgrade the Greek educational system (Owusu-Boampong \& Holmberg, 2015). Incorporating synchronous and asynchronous technologies in formal educational settings was also an important priority due to Greece's geographical particularities, whose many islands and mountains make access to important locations challenging. However, a specific strategic plan was never set for the coherent cultivation of distance education within Greek universities and almost all of them keep offering only face-to-face courses. Before the pandemic, the only actions related to the implementation of distance education in Greece were related to the use of asynchronous platforms (e.g., the Open eClass) or teleconferencing technologies as means for enhancing or supporting face-toface undergraduate or postgraduate courses (Anastasiades, 2014; Anastasiades \& Kotsidis, 2013).

Having in mind the possibility of an extended and more widespread adoption of distance education across universities for the months to come, our aim in this paper is to identify undergraduates' difficulties with online learning. In particular, we aim at answering the following research questions:

- RQ1. What barriers did undergraduates encounter during their transition to online learning?

- RQ2. What can be inferred about the quality of online learning as it was implemented during this transition?

To that end, we conducted a qualitative in nature study which involved surveying more than 2000 undergraduates from Greek universities. The paper is structured as follows: first, we present our synthesis of the literature pertaining to undergraduates' barriers to online learning; second, we outline the theoretical framework used in our study (theory of Transactional Distance, deep and meaningful learning); third, we describe in detail our methodological approach (online questionnaires for gathering data, analyzing undergraduates' responses in a qualitative manner, evaluating the quality of our inferences); fourth, we present our results in the form of our coding frame, and; finally, we position our findings within the wider literature and present our conclusions.

\section{Literature Review}

Bernard et al.'s (2019) systematic literature review on online learning since 2000, offers a good starting point in developing an understanding about the state of the art. By using their work as a basis, we conducted our review and we have noticed that the student perspective 
is not well articulated in the literature. The lack of literature offering an overview of undergraduates' experiences in online settings has been noted by early (e.g.Howland \& Moore, 2002) and more recent investigations (e.g.Blackmon \& Major, 2012). Given our research questions, our aim in this section is to present a number of selected studies reporting on the difficulties undergraduates face while engaged in online learning programs. In the following paragraphs, the terms barriers, difficulties, issues, challenges, limitations and obstacles are used interchangeably in order to refer to factors limiting undergraduates' participation in online learning.

Muilenburg and Berge (2005) surveyed a large sample $(N=1056)$ of students participating in various online programs about the difficulties they face during their studies. By following an exploratory factor analysis approach, students' barriers to online learning were categorized into eight factors: (1) administrative and instructor issues (course materials not always delivered on time, instructors' lack of knowledge in teaching online, lack of timely feedback from instructors, not appropriate class size, lack of sufficient academic advisors online); (2) social interactions (lack of student collaboration, lack of social context cues, students' feeling of being afraid or isolated in online courses); (3) academic skills (students' lack of skills in areas such as writing, reading and communication); (4) technical skills (students' unfamiliarity with online technical tools, lack of online software skills, fear of using new tools); (5) learner motivation (students' lack of personal motivation for online learning, procrastination, increased responsibilities, online environments not inherently motivating); (6) time and support for studies (lack of time or support from family and friends, insufficient time, significant interruptions during study at home/work); (7) cost and access to the internet (lack of adequate internet access or access to needed technology) and; (8) technical problems (lack of consistent platforms, browsers, and software, lack of technical assistance).

Appana (2008) notes that although online learning is a worthwhile investment for institutions, the literature highlights several limitations that must be overcome from both the student's and the instructor's point of view. By building upon Bartolic-Zlomislic and Bates' (1999) work and relevant literature, Appana (2008) proposes several limitations of online learning. These include barriers such as funding, adequate time, organizational preparedness, student readiness, differing stages of team development, crisis management, faculty learning curve, limited language skills, technical support, team effort, synchronous or asynchronous approaches, costs, accessibility of course materials, delayed feedback and evaluation, and assessment. Those concerning students, relate to the perceived time-consuming nature of online learning, the necessity of having access to a computer and the internet, the burden of dealing with technical difficulties and delays that may occur during an online lesson, the self-directed nature of online learning, cultural and language differences among students, delays in receiving feedback from instructors and the distractions created by the internet itself.

Blackmon and Major (2012) note that although our understanding about students' characteristics and outcomes in online courses is well developed, less in known about their overall experiences in such environments. By conducting a literature review, the authors identified the presence of five factors influencing undergraduates' experiences in online courses. These are organized into factors stemming from students' behavior and factors arising from an instructor's behavior. The factors concerning students include issues related to the need for balancing between educational commitments and family life; the ability to successfully manage their time and organize accordingly their schedule and; the necessity of making a personal commitment in terms of accepting the responsibility and autonomy required when enrolled in an online course. The choices instructors make also influence 
students' experience in online learning. These concern the accessibility of the instructor i.e., building intellective relationships with students and creating opportunities for students to connect i.e., creating a sense of community in the e-class.

Kassandrinou et al. (2014) analyzed data from interviews with 12 postgraduates attending a blended online setting in order to identify the factors influencing the interactions among students. Students reported a variety of barriers such as geographical distance, overcharged schedules, lack of spare time, the reserved personality of some students or the instructor's inability in encouraging communication and cooperation among them.

Kebritchi et al. (2017) note that although many empirical studies have been conducted in undergraduate online courses, few of them offer a synthesis on the challenges faced in such environments. By conducting a systematic literature review, Kebritchi et al. identified challenges related to learners, instructors and content development. Challenges related to learners include undergraduates' expectations, their readiness in attending an online course (e.g., required learning styles and technical skills) and also include sociocultural aspects of their participation (e.g., sense of belonging to a community). Instructors' challenges concern the multiple roles faculty members often adopt when teaching online (e.g., choosing appropriate teaching methods, establishing social relations with students or managing administrative, organizational and technical tasks); the transition to online teaching (e.g., limited number of verbal and non-verbal interactions with students, adjusting the lecture format, lack of interest in teaching online, lack of appropriate training); time demands when preparing and delivering online courses, and; adopting an effective teaching style (e.g., using certain e-learning methods and strategies). Finally, issues related to content include instructors' role in content development (e.g., not participating in content development, challenges in adapting face-to-face material for online teaching); the integration of multimedia (e.g., identifying the right kind and amount of multimedia to be used); the implementation of instructional strategies in content development (e.g., determine suitable activities to be included in a course) and; the organization and assessment of a course's content (e.g., dividing content into meaningful sections, incorporating both formative and summative assessment).

Kara (2020) analyzed qualitative data from 20 undergraduates, regarding their views about the lecturer, the instructional methods and materials used in an online course. Kara's analysis showed that a number of barriers such as conflicts between work and lesson schedules, a class' size or a lecturer's inability to develop interactive online material, limit undergraduates' learning experience.

Joshi et al. (2020) report on the barriers of online learning from both an instructor and student perspective. By following a Strengths, Weaknesses, Opportunities and Threats (SWOT) approach, Joshi et al. reviewed the literature in order to design a survey instrument. The negative aspects of online learning were organized into two factors (weaknesses and threats), with each factor containing four categories of barriers. Weaknesses include limitations such as the diminished quality of instruction in online learning environments, the lack of social interactions, delays in feedback from instructors and the lack of hands-on experience. Threats are related to dangers that may occur when implementing online learning such as the possibility of challenging a university's prestige in the long-run, the vulnerability to scams or academic dishonesty, the possibility of creating obstacles for innovative ideas and the societal skepticism on the quality of online education. Joshi et al. administered the survey to agriculture and natural resource science students and instructors in a US-based university in two rounds ( $N=65$ and $N=56$ respectively). Results showed that students consider the lack of hands-on experience and the diminished quality of instruction as the most important weaknesses of online learning, whereas the vulnerability to scams 
or academic dishonesty and the societal skepticism on the quality of online education were viewed as the most significant threats.

The literature reviewed in this section can be divided into empirical studies (Joshi et al., 2020; Kara, 2020; Kassandrinou et al., 2014; Muilenburg \& Berge, 2005;) and literature reviews (Appana, 2008; Blackmon \& Major, 2012; Kebritchi et al., 2017). In a nutshell, undergraduates have been found facing barriers related to: (1) the technical problems occurring during synchronous or asynchronous online sessions (Appana, 2008; Muilenburg \& Berge, 2005); (2) the limited social interactions that online environments can afford (Joshi et al., 2020; Kassandrinou et al., 2014; Kebritchi et al., 2017; Muilenburg \& Berge, 2005); (3) the struggle in balancing work/family and education commitments (Blackmon \& Major, 2012; Kara, 2020) and; (4) the lack of the necessary competencies in teaching (Blackmon \& Major, 2012; Joshi et al., 2020; Kara, 2020; Kebritchi et al., 2017; Muilenburg \& Berge, 2005) or learning in a distance education environments (Appana, 2008; Kebritchi et al., 2017; Muilenburg \& Berge, 2005). Although previous research has attempted to understand how undergraduates experience online learning, our sense of the field aligns with comments from earlier studies and systematic reviews which underline a lack of literature focusing on the student perspective (e.g., Blackmon \& Major, 2012; Howland \& Moore, 2002) and exhibit a dearth of empirical reports from countries where distance education is not a well-established didactical approach (Bernard et al., 2019; Kebritchi et al., 2017), as it is the case of Greece.

\section{Theoretical Framework}

\subsection{Theory of Transactional Distance}

Among the most widely used frameworks in studying distance education is Moore's theory of Transactional Distance (TD) (Moore, 1993). The core idea of Moore's theory is that the distance between instructors and learners is not geographical but rather pedagogical in nature (Moore \& Kearsley, 2011). Within this framework, the space created by the spatial and/or temporal separation is called transactional distance and it refers to the psychological and communication gap between teachers' and learners' behaviors (Moore, 1993). According to Moore (1993), the extend of transactional distance is a function of three clusters of variables: dialogue, structure and learner autonomy.

The term dialogue refers to "an interaction or series of interactions having positive qualities that other interactions might not have. [...] The direction of the dialogue in an educational relationship is towards the improved understanding of the student." (Moore, 1993, p. 21). The extent and nature of dialogue is determined by several factors such as the educational philosophy of those responsible for the design of a course, teachers and learners' personalities, the subject-matter of the course as well as by environmental factors related to the medium used for communicating, the number of students participating in a course and the physical environment of both the teachers and learners (Moore \& Kearsley, 2011). When dialogue is low, transactional distance increases. Structure refers to elements found in a course's design (e.g., educational objectives, teaching strategies, evaluation methods) and expresses how rigid or flexible these elements are in relation to each learner's individual needs (Moore \& Kearsley, 2011). Structure is determined by the medium used for communicating, teachers' philosophy and emotional characteristics, learners' personalities as well as by institutional constraints (Moore, 1993). When structure is high, transactional 
distance increases. Finally, the concept of learner autonomy takes into consideration the different capacities each student has for making decisions regarding her own learning and thus describes the extent to which a learner can exert control and manage her learning experiences (Moore \& Kearsley, 2011). Learner autonomy is dependent on transactional distance: "the greater the transactional distance is the more responsibility the learner has to exercise" (Moore \& Kearsley, 2011).

\subsection{Types of Interaction}

Moore (1989) suggested that at least three types of interactions take place in distance education programs: learner-content (LC), learner-instructor (LI) and learner-learner (LL). Learner-content interaction refers to the interaction between the learner and the subject of study. This type of interaction aims at improving a learner's understanding and perspective on a topic via the use of various types of media prepared by an instructor. Learnerinstructor interaction relates to the interaction taking place between "the learner and the expert who prepared the subject material, or some other expert acting as instructor" (p. 2). Moore (1989) noted that a learner is influenced more when learner-instructor interaction is added to the educational process since he/she is able to "draw on the experience of the professional to interact with the content in [a] manner that is most effective for that particular individual learner" (p. 3). Finally, learner-learner interaction refers to the interactions taking place between students in distance education environments.

Advances in the technologies used in distance education led many scholars to suggest additional forms of interactions in online environments. One such expanded version was suggested by Anderson and Garrison (1998). Anderson and Garrison proposed that three additional forms of transactional interactions should be added into Moore's initial model: instructor-instructor (referring to the communication between teachers e.g. for exchanging knowledge), instructor-content (concerning the process of creating and designing instructional material) and content-content interactions (pertaining to intelligent agents i.e. software components that can perform various tasks without the need for constant human intervention).

Although TD is a well-recognized theory in the field, many authors consider that it is not well synchronized with current practices and lacks a proper social component (Kang \& Gyorke, 2008). Such criticisms might be related to the several attempts made so far in making TD a more realistic model and include the addition of learner-interface (Hillman et al., 1994) and learner-environment (Burnham \& Walden, 1997) interactions.

\subsection{Deep and Meaningful Learning}

The importance of facilitating interactions among and between learners, instructors and content in distance education has been well articulated in the literature. Moore (1989) recommended that courses should be organized in ways that both ensure the "maximum effectiveness of each type of interaction" and "the type of interaction that is most suitable for the various teaching tasks of different subject areas, and for learners at different stages of development" (p.5). In more recent years, Anderson (2003a) developed his "equivalency theorem" which offered a slightly different but more refined approach on supporting interactions in distance education programs (Bernard et al., 2009). According to the "equivalency theorem" (Anderson, 2003a): 
Deep and meaningful formal learning is supported as long as one of the three forms of interaction (student-teacher; student-student; student-content) is at a high level. The other two may be offered at minimal levels, or even eliminated without degrading the educational experience. High levels of more than one of these three modes will likely provide a more satisfying educational experience, though these experiences may not be as cost or time effective as less interactive learning sequences. (p. 4)

For Anderson (2003a), the "equivalency theorem" implies that similar educational outcomes can be achieved by substituting one type of interaction with one of the others, as long as the interactivity levels are the same.

\subsection{Ideas Incorporated in this Study}

Given this study's focus, we have employed certain aspects from the theory of Transactional Distance (types of interaction, deep and meaningful learning) as a way of analyzing our data and interpreting results. The types of interaction acknowledged by** Anderson and Garrison's model (1998) were used as the basis for positioning the identified in the data barriers between learners (LL interaction), learners and their instructor (LI interaction) and learners and content (LC interactions). Barriers were then interpreted either as factors limiting certain interactions or as consequences of the limited interactivity characterizing LL, LI or LC interactions. Following that, we inferred whether the interactions acknowledged by Anderson (2003a) as being particularly important for supporting undergraduates' deep and meaningful learning (LL, LI, LC) were at a high level. This approach enabled us to infer about the quality of online learning as it was implemented by Greek universities during the pandemic.

\section{Methodology}

The present study follows a qualitative design and as such it aims at providing a detailed understanding of participants' views on a topic, while considering that reality is socially constructed and multiple in nature (Lincoln \& Guba, 1985). Our data are qualitative in nature and were obtained by following a convenience sampling strategy. This involved surveying large cohorts of Greek undergraduates about the difficulties they faced during their transition to a fully online environment.

\subsection{Context of Study}

All universities in Greece are tuition-free and they operate under the authority of the Ministry of Culture, Education and Religious Affairs. Students are admitted to universities after succeeding in wide-state qualifying exams and almost all of them are white and have a Greek citizenship. Greece has over 30 universities with teaching being primarily in Greek, although many programs (especially at the postgraduate level) are offered in other languages as well (most commonly in English). Prior to the pandemic, the only university in Greece offering fully online undergraduate and postgraduate programs was the Hellenic Open University. 
Due to the COVID-19 pandemic, all educational institutions stopped operating on the 11th of March 2020, however, from the 16th of March 2020 all Greek universities resumed their classes in a fully online manner for the remaining semester. The Ministry of Culture, Education and Religious Affairs allowed universities to choose whether they will proceed in a synchronous or asynchronous manner, so each institution adopted a different approach. The present study took place during this period.

\subsection{Participants and Recruitment Process}

All participants were recruited through university emailing lists which were obtained after contacting students' unions from all Greek universities. In the email inviting undergraduates to participate in our study, we shortly informed them about the rationale of our study, the voluntary and anonymous nature of their participation, the duration of the survey (approximately $10 \mathrm{~min}$ ) and they were also provided with a link in order to access our web-based questionnaire. No incentives were provided to students for their participation in the survey. In total, 2093 undergraduates all registered at various Greek universities participated in our survey. The majority of them were from the Hellenic Mediterranean University (15.24\%), the University of Crete (14.91\%), Democritus University of Thrace (14.86\%), the University of West Attica (9.27\%), the University of Patras (8.89\%), Aristotle University of Thessaloniki (7.45\%), National and Kapodistrian University of Athens (5.64\%) and the University of Ioannina (3.34\%).

\subsection{The Survey}

Due to the restrictions posed during the first lockdown period in Greece, a survey approach methodology was adopted. An initial version of the questionnaire inspired by Muilenburg and Berge's (2005) survey was written by the second author. Our main intention was to collect data regarding undergraduates' perceived barriers to online learning, however a few other items were also included as means for gaining a better understanding of our sample. A team of qualified experts from the Hellenic Mediterranean University (HMU) reviewed all questions for accuracy, grammatical errors and consistency. The questionnaire was then piloted with a small sample (30) of postgraduate students from HMU and after taking into account their suggestions, a final version was designed by using Google Forms.

The survey was enabled to accept responses on the 1st of May 2020 and was deactivated on the 30th of June, since the last response was received on the 6th of June 2020. The questionnaire included seven items (Table 1): questions 1-6 were dichotomous while question 7 was open-ended. These were related to how quickly undergraduates adapted to the new situation (Q1); their past experience with online learning (Q2); the perceived effectiveness of online learning (Q3, Q4, Q5); undergraduates' confidence with online learning technology (Q6) and; the perceived limitations of online learning (Q7). In order to make our survey short and increase the response rate, no personal or demographic data (e.g., name, gender, year of studies, subject specialization) were collected.

\subsection{Method of Analysis}

In analyzing questions 1-6, descriptive statistics were used. For question seven, Qualitative Content Analysis (QCA) was used. QCA is a method for describing in a systematic 
Table 1 The survey questions

\begin{tabular}{ll}
\hline Question & Type (Responses) \\
\hline Q1: Since your institution's reopening, have you adjusted to online learning? & Closed (Yes, No) \\
Q2: Do you have any previous experience with online learning? & Closed (Yes, No) \\
Q3: Are you satisfied with the learning effectiveness of the online platform used by & Closed (Yes, No) \\
your institution? & \\
Q4: Are you satisfied with the learning effectiveness of your lecturers' online teaching? & Closed (Yes, No) \\
Q5: Has the material from current semester's curriculum been covered in time? & Closed (Yes, No) \\
$\begin{array}{ll}\text { Q6: Are you confident with your skills in using online learning technology? } \\
\text { Q7: Please mention a few issues (2-3) that you are currently experiencing with online }\end{array}$ & Open-ended \\
learning (if any) & \\
\hline
\end{tabular}

All items are translated from Greek

way the meaning of qualitative material (Schreier, 2014). Contemporary QCA is similar to thematic analysis but it has distinct features when compared to other qualitative approaches such as Thematic Analysis or Grounded Theory (Drisko \& Maschi, 2016). At the core of QCA lies the coding frame, aiming at classifying all data as instances of a set of aspects related to the research question(s). It has a hierarchical structure and consists of at least two levels: the main category or categories (the aspects on which a researcher wants to focus his/her analysis) and the subcategories (instances of what is said about the main categories) (Schreier, 2014). In this sense, the main categories behave like variables related to certain aspects of a phenomenon and subcategories are the values of these variables.

Following Schreier (2014), our coding frame was built in a series of steps. First, all relevant material (i.e., responses to Q7) was selected and stored in one text file. Second, the coding frame's main category was generated in a concept-driven manner i.e., one main category reflecting undergraduates' barriers to online learning was created. Following that, the subcategories i.e., examples of barriers, were generated. This was done by either adopting a concept-driven approach i.e., based on Muilenburg and Berge's (2005) work or by following a data-driven strategy i.e., by either identifying a theme (subsumption) or paraphrasing passages (successive summarizing). Then, each subcategory was given an appropriate name, a description, a set of conditions under which a response could be coded under a certain subcategory and a representative example. This process was repeated several times until all material was coded and no further revision was required. Due to this highly systematic procedure, we were able to generate a coding frame that enabled us to associate the contents of each subcategory with the theoretical constructs used in our study.

In order to evaluate and enhance the quality of our inferences, we followed a number of techniques as suggested by Lincoln and Guba (1985), Schreier (2014) and Teddlie and Tashakkori (2009). The survey data were split into six parts and every co-author was assigned with two parts (roughly 700 responses). Each author analyzed one part and the generated coding frames and inferences were compared (triangulation of investigators). The coding was performed by using the R package RQDA (Ronggui, 2016). This process was repeated on the second half of the data and inferences from both parts were compared (referential adequacy). This led to a revised version of our coding frame and set of inferences which were then assessed for their credibility by the same team of experts who evaluated our questionnaire (peer debriefing). In addition to the above, the final version of the coding frame was also assessed in terms of its unidimensionality (ensuring that each 
category captures only one aspect of the material); mutual exclusiveness (ensuring that each coded segment was assigned to only one main category) and; exhaustiveness (ensuring that all relevant material was coded).

\section{Findings}

\subsection{Descriptive Findings}

In total, 2093 undergraduates participated in our survey. Descriptive statistics for questions 1-6 are presented at Fig. 1. Although the majority of participants (82.4\%) reported not having any previous experience with distance learning, over half of them $(68.6 \%)$ stated that they have adjusted to the new environment. In terms of the platform's perceived learning effectiveness and lecturers' online teaching, most of the students reported being satisfied ( 68.2 and $73.8 \%$ respectively). Similarly, the majority of students in our sample (73.9\%) reported that all the learning material has been covered according to current semester's curriculum. Finally, only half of the students (53.5\%) reported being confident with using online learning technologies.

\subsection{Barriers to Online Learning}

In this section we report on our analysis of students' responses regarding their problems during the transition to online learning (Q7). In total, 93\% $(N=1943)$ of students in our sample answered Q7. The majority of them (87\%) reported facing some kind of an issue with online learning, while the rest reported not having any problems at all. Given that we specifically asked undergraduates to state in Q7 two or three issues they are currently experiencing, each answer to Q7 would yield up to three responses that could be possibly coded as barriers or a maximum of $1943 \times 3=5829$ responses altogether. In our data, we identified 3022 responses (not necessarily from different students). Of them, 2,608 were classified as barriers and were assigned to a subcategory of our coding frame (Fig. 2, Table 2), 258 were from students reporting having no issues with online learning (these were simple responses in the form of "No problems at all" or "Everything is fine"), whereas 156 included negative comments for online learning when compared with face-to-face instruction e.g. "Not being physically present has negative consequences in many areas of a student's life" (S.126) or "The overall experience is not as enjoyable as in face-to-face learning." (S.68).

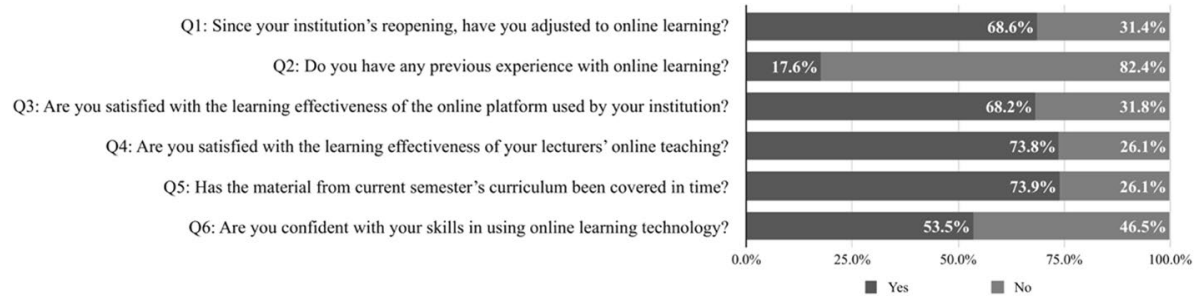

Fig. 1 Undergraduates' responses regarding how quickly they adapted to online learning (Q1), previous experience with online learning (Q2), the perceived learning effectiveness of online learning (Q3-Q5) and confidence when using online learning technology (Q6) 


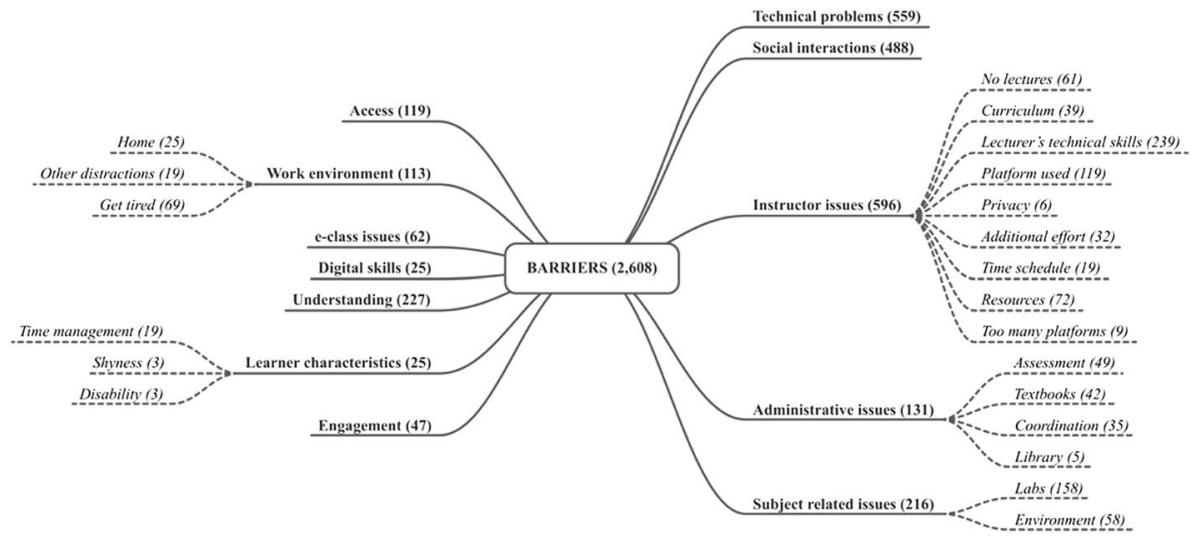

Fig. 2 Coding frame for student barriers to online learning. Numbers in parentheses indicate coding frequencies

Our coding frame consists of 12 subcategories, i.e., 12 different types of perceived barriers, with one category including different types of barriers with low frequencies that did not fit to other subcategories. The generated subcategories were (Fig. 2, Table 2):

(1) Technical problems: internet connection issues occurring during an online lecture.

(2) Social interactions: the perceived limited social interactions that a synchronous environment can afford or restrictions when collaborating online for a group assignment.

(3) Instructor issues: problems that undergraduates perceived as being caused by their lecturers. This subcategory was further divided into nine sub-subcategories: not using synchronous methods i.e. online lectures (no lectures); adopting a fast pace in covering the curriculum (curriculum); a lecturer's unfamiliarity with technology or using an online environment to teach (lecturers' technical skills); using teleconferencing platforms (e.g., Skype for Business) that permit a certain number of participants (platform used); sudden increase in workload (additional effort); inconsistency in timetables e.g. lectures taking place during weekends (time schedule); not uploading resources in the university's virtual learning environment (resources); confusion caused by the excessive number of different platforms used by lecturers (too many platforms) and; the strict privacy rules pertaining to recording an online lecture (privacy).

(4) Administrative issues: problems that undergraduates perceive as being caused by administrators. This subcategory was further divided into four sub-subcategories: lack of coordination or announcements (coordination); textbooks not delivered on time (textbooks); lack of updates regarding the forthcoming exams (assessment) and; not having access to material from the library (library).

(5) Subject-related issues: the perceived limited affordances of online environments in relation to certain subjects of study such as Mathematics, Music, Physics, Medicine and Engineering. This subcategory was further divided into two sub-subcategories: issues related to the practice of teaching and learning in a physical environment (environment) and; not being able to conduct work in a lab (labs).

(6) Access: not having access to online learning e.g., not owning a laptop or having access to an internet connection. 
Table 2 The subcategories of our coding frame (left column) and examples of undergraduates' responses to Q7 ("Please mention a few issues (2-3) that you are currently experiencing with online learning (if any).") that were assigned to each subcategory (right column)

\begin{tabular}{ll}
\hline Subcategory & Quotes \\
\hline Technical problems & $\begin{array}{c}\text { "Poor quality of my internet connection, with constant disruptions of video and } \\
\text { sound." (S.378) }\end{array}$ \\
Social interactions & $\begin{array}{l}\text { "There is almost no discussion taking place, it's difficult to ask a question." } \\
\text { (S.1057) }\end{array}$
\end{tabular}

Instructor issues

Administrative issues

Resources: "Some lecturers do not upload material from their presentations. Not all lectures are video-recorded so there is no asynchronous material..." (S.249)

No lectures: "Specifically, in our institution, not all lecturers use synchronous methods. They just upload some notes on the e-class platform... In this case, students' questions are not adequately covered [when] compared to the physical presence [sic.] or even with the correct use of online learning." (S.937)

Platform used: "The platform used by my lecturer (Skype for Business) is insufficient for such a large number of students. When the limit of 250 participants is reached, some students cannot attend and they stay out of the learning process." (S.651)

Curriculum: "Because of this way of teaching, many of our lecturers cover much faster material than they would in the normal way." (S.589)

Privacy: "Some lecturers do not record online lectures due to data protection regulations." (S.1216)

Time schedule: "...inappropriate class hours e.g., Saturdays, Sundays or 8 pm daily." (S.1478)

Additional effort: "Many lecturers believe that the 'new reality' has given us extra time and they burden an already burdensome curriculum with additional requirements." (S.522)

Lecturer's technical skills: "Many lecturers do not know how to use certain technologies and they just teach us by simply reading the slides they upload without interacting with the students, so the lesson becomes quite boring." (S.1628)

Coordination: "Most of my complaints concern mainly my university, which was late in mobilizing both at the beginning of the semester and before the measures were taken, as well as after..." (S.950)

Textbooks: "The lack of books in some courses makes it impossible to attend." (S.1891)

Exams: "We do not know when the exams will start." (S.493)

Subject-related issues Labs: "Laboratory lessons end up being theoretical as there is no physical presence and contact with the experiments." (S.814)

Environment: "I study Physics. Being able to show my solution to my lecturer is often necessary but it becomes significantly more difficult and time consuming with distance education. The same applies for teaching itself. Seeing the equations written [by my lecturer] 'live' and explaining their meaning at the same time is essential to my understanding." (S.682)

Access

Work environment

"Many of us do not have the necessary equipment for participating in a class e.g., microphone, camera or a computer that easily handle the programs used." (S.81)

Other distractions: "Distraction from environmental factors that I cannot always control." (S.1975)

Home: "There are times when I forget about the lecture, I watch something else on the internet because there is no one there to supervise me and I have the comfort of my own home". (S.94)

Get tired: "We get tired when attending lectures in front of a computer for many hours." (S.87)

e-class issues

"Some students take advantage of the platforms used in order to make fun during a class or they interrupt more often the lecturer." (S.1790)

Digital skills "I find it difficult to follow the instructions of using the e-class platform. Therefore, I do attend online classes, I only access my lecturer's notes." (S.1085) 
Table 2 (continued)

\begin{tabular}{ll}
\hline Subcategory & Quotes \\
\hline Understanding & $\begin{array}{c}\text { "I have difficulties understanding the material taught online as opposed to a normal } \\
\text { lecture." (S.340) }\end{array}$ \\
Learner characteristics & $\begin{array}{c}\text { Time management: "I have difficulties in planning my study. This is because the } \\
\text { lectures are recorded and I often think it doesn't matter I will watch it tomorrow" } \\
\text { and eventually I watch it after a week." (S.48) } \\
\text { Shyness: "I do not feel comfortable talking or asking questions etc." (S.37) } \\
\text { Disability: "Some of us have a visual or auditory impairment thus we cannot par- } \\
\text { ticipate in online learning." (S.142) } \\
\text { "With distance education I have lost my courage to participate in the course." } \\
\text { (S.1250) }\end{array}$ \\
Engagement
\end{tabular}

All quotes are translations from Greek and for each student an alphameric code has been used (S.1: student 1, S.2: student 2 etc.). If present, the name of the corresponding sub-subcategory precedes the quote

(7) Work environment: problems related to a student's working environment. This subcategory was further divided into three sub-subcategories: issues occurring due to working from a home environment (home); distractions caused due to the presence of digital devices such as the computer itself or other mobile devices (other distractions) and; getting tired because of working many hours in front of a computer screen (get tired).

(8) e-class issues: problems occurring during an online lecture such as accidents with open microphones and disruptions caused by background noises or undergraduates not following the norms of a class.

(9) Digital skills: barriers related to undergraduates' unfamiliarity with technologies used in online learning.

(10) Understanding: the perceived difficulty in understanding the material taught in online lectures or via the resources provided by the lecturer.

(11) Learner characteristics: barriers related to undergraduates' characteristics that could affect their engagement with online learning. This subcategory was further divided into three sub-subcategories: having difficulties in properly managing time or attending regularly online lectures (time management); feeling uncomfortable in asking questions during a lecture (shyness) and; barriers related to a student's physical/ sensory disabilities (disability).

(12) Engagement: this barrier relates to undergraduates' perception that participating in online learning is not inherently motivating especially when compared to face-to-face environments.

As we can see from Fig. 2, the most frequently reported barriers include problems that undergraduates perceived as being caused by their lecturers (instructor issues: $22.85 \%$ ), internet connection issues (technical problems: $21.43 \%$ ) and the perceived limited social interactions that a synchronous environment can afford (social interactions: $18.71 \%$ ). These were followed by difficulties in understanding the material taught (understanding: $8.70 \%$ ) or the limited affordances of online environments in relation to certain subjects of study (subject related issues: $8.28 \%$ ). 


\subsubsection{Restrictions Among Interactions}

Having in mind the interactions that take place in online environments, we interpreted some of the barriers from our QCA analysis as factors restricting certain interactions (i.e., barriers that influence an interaction), and others as indicators of low interactivity (i.e., barriers that can be considered as results of limited interactions among components). The operationalization of Moore's (1989) or Anderson and Garrison's (1998) models as a way of identifying factors affecting interactions in distance education, has been adopted by many studies. For example, Kara (2020) interpreted the barriers identified in his study as factors affecting learner-instructor and learner-content interactions. Similarly, Kassandrinou et al. (2014) characterized barriers derived from their analysis as factors restricting learner-learner interaction, which consequently result in increased transactional distance among postgraduates. This approach has been also used in the design of surveys for quantifying transactional distance: for instance, Paul et al. (2015) developed a revised scale for measuring transactional distance among three types of interaction: learner-learner, learner-instructor and learner-content.

In our data, we identified factors restricting learner-learner (LL), learner-content (LC) and learner-instructor (LI) interactions. As factors restricting LL, LC and LI interactions we have classified barriers related to the necessary technologies for participating in online learning, a student's working environment and those concerned with undergraduates' personal characteristics. This is because, as Anderson (2003b) notes, "all forms of interaction in a distance education context are, by definition, mediated forms of interaction" (p.132). Moreover, barriers related to a student's workplace interferes when interacting with peers, the instructor or the content of a course (Burnham \& Walden, 1997). Finally, personal characteristics (e.g., not managing time properly) can potentially affect an undergraduate's experience with online learning. Thus, barriers designated as restricting LL, LC and LI interactions include connection issues occurring during an online lecture (technical problems), not having access to online learning (access), undergraduates' unfamiliarity with technologies used in online learning (digital skills), an undergraduate's personal characteristics (learner characteristics) as well as problems related to a student's working environment (work environment).

Barriers restricting the LC interaction refer to issues that generally inhibit students from having access to educational material or limit in some way undergraduates' learning experience. In this category we have included barriers that undergraduates perceived as being caused by their lecturers (instructor issues) and administrators (administrative issues). These include not having access to a number of resources and services (textbooks, library, resources, no lectures), lack of coordination and updates (coordination, assessment) or changes in the way a lecturer teaches (curriculum, additional effort, time schedule, too many platforms). Barriers restricting IC interaction refer to problems complicating the instructional design process and include a lecturer's unfamiliarity with technology or online environments (instructor issues: lecturer's technical skills).

Barriers not classified as restricting interactions were considered as results of the limited interactivity characterizing LL or LI interactions. The perceived limited social interactions that synchronous and asynchronous environments can afford (social interactions) and problems occurring during an online lecture (e-class issues), were considered as indicators of low interactivity between learners (LL) and learners with their instructor (LI). Undergraduates' view that online environments are limited in relation to certain subjects of study (subject-related issues) and perceived difficulty in understanding the 
material taught in online lectures (understanding) were considered as indicators of low interactivity between learners and content (LC). Finally, undergraduates' perception that participating in online learning is not inherently motivating (engagement) was treated as an indicator of the overall low interactivity characterizing LL, LI and LC interactions since students' ability to communicate their ideas among their peers and their instructor (Anderson, 2003b) as well as the capacity for interacting with the content of their study (Anderson, 2003a), increase their motivation towards deep and meaningful learning.

\section{Discussion and Conclusion}

Arguably, the forced transition to online learning due to the COVID-19 pandemic posed great challenges to all universities around the world. Our aim in this paper was to identify the barriers that undergraduates encountered during this transition and make inferences about the quality of the way online learning was implemented in Greece. Our sample $(N=2093)$ consisted mostly of students not having any previous experience with online learning $(82.4 \%)$, with only half of the total sample (53.5\%) being confident in using the necessary technologies for participating in online learning. Despite that, the majority of students reported having adjusted to the new reality $(68.6 \%)$ and expressed their satisfaction regarding the platform used (68.2\%), their lecturer's teaching $(73.8 \%)$ or the material covered from the semester's curriculum (73.9\%).

Although the above results suggest a relatively uncomplicated transition, undergraduates' responses regarding the issues they faced offered a more detailed account of their experience. By following a QCA approach and based on Muilenburg and Berge's (2005) work, we classified undergraduates' barriers to online learning into 13 categories. The most frequently reported barriers include problems that undergraduates perceived as being caused by their lecturers (instructor issues: $22.85 \%$ ), internet connection issues occurring during an online lecture (technical problems: 21.43\%) and the perceived limited social interactions that a synchronous environment can afford (social interactions: 18.71\%). Other less frequently reported barriers included difficulties in understanding the material taught (understanding: $8.70 \%$ ) and the limited affordances of online environments in relation to certain subjects of study (subject related issues: $8.28 \%$ ).

The barriers identified in this study reflect those reported in the literature before the pandemic: for example, technical problems (Appana, 2008; Muilenburg \& Berge, 2005), the lack of social interactions in online environments (Joshi et al., 2020; Kara, 2020; Kassandrinou et al., 2014; Muilenburg \& Berge, 2005), the limited affordances of online environments in relation to certain subjects (Joshi et al., 2020) or problems stemming from an instructor's approach in designing and organizing an online course (Blackmon \& Major, 2012; Kara, 2020; Muilenburg \& Berge, 2005) are among the difficulties that have been reported during the last 15 years by various studies. In addition, our findings are in agreement with studies conducted amid the pandemic and concern barriers related to technical problems with the internet, undergraduates' environment (e.g., working from home), not having access to laboratories and equipment or limited social interactions between peers (Chakraborty et al., 2021; Mishra et al., 2020; Patricia Aguilera-Hermida, 2020). It is worth noting that although Greek universities rely almost exclusively to face-to-face instruction with no prior experience in delivering course content in a fully online manner, the barriers identified in our paper share many similarities with studies conducted in countries with different educational systems and/or traditions in online learning. 
By incorporating learner-learner (LL), learner-instructor (LI) and learner-content (LC) interactions (Anderson \& Garrison, 1998; Moore, 1989) into our analysis, we were able to go beyond a descriptive account of undergraduates' barriers and characterize some of them as factors limiting certain interactions and others as results of these restricted interactions. Barriers characterized as factors restricting LL, LI and LC interactions include: internet connection issues (technical problems); not having access to online learning (access); problems related to a student's working environment (work environment); undergraduates' unfamiliarity with technologies (digital skills) and their personal characteristics (learner characteristics e.g., time management). Barriers characterized as factors limiting LC interaction include problems that undergraduates perceived as being caused by their lecturers (instructor issues) or by administrators (administrative issues e.g., textbooks availability). The rest of the barriers were considered as a result of the overall limited interactivity characterizing LL, LI and LC interactions (engagement), in LL or LI interactions (social interactions, e-class issues) and in LC interaction (understanding, subject related issues).

Given that deep and meaningful learning can be supported as long as one of the LL, LI or LC interactions are retained at a high level (Anderson, 2003a), our analysis suggests that the way online learning was implemented during the COVID-19 pandemic by Greek universities did not result a meaningful learning experience for undergraduates. This is because none of the LL, LI and LC interactions were at particularly high levels: undergraduates characterized their online learning as an experience mainly marked by limited social interactions (LL, LI) and inadequate access to synchronous or asynchronous learning material (LC). From a TD perspective, limited social interactions can be considered as an indication of the increased transactional distance among learners and between learners and their instructor, whereas barriers related to understanding can be treated as indicators to an increase in the transactional distance between learners and their content of study. Our aim in this paper was not to quantify the interactivity between LL, LI or LC components and our analysis does not imply any causal relationship between barriers and certain interactions. In addition, due to our methodological approach, our study has its limitations, which do not allow us to make any generalizations.

Arguably, the pandemic impacted heavily the higher education sector and acted as the trigger for realizing the importance and potential of distance education. We hold the view that the role of universities is crucial in attaining the shortcomings of online learning in both the pandemic and post-pandemic period. Conceptualizing online learning environments as a set of interactions between instructors, learners and content (LI, LL, LC) that must be kept at high levels, can be both used as a pedagogical device and a set of recommendations that can inform and guide the practice of online learning. This can be mainly achieved by providing training to the main actors involved in the learning process, i.e., instructors and students. Professional development for instructors could focus on equipping instructors with the necessary digital skills and appropriate pedagogical knowledge for delivering effectively content and enabling social interactions among learners or between learners and their instructor. Such courses could include training on using various synchronous and asynchronous technologies (e.g., cloud sharing, videoconferencing, electronic voting systems, online assessment tools) and pedagogies that promote active and collaborative learning (e.g., flipped or blended approaches). Training courses for students might concentrate their efforts on developing the required digital skills for participating in an online course but more importantly cultivating students' autonomy as learners. Such courses could aim at training students on managing effectively their time, scheduling their study hours and overall adopting a self-directed approach to learning. 
Funding Not applicable.

Availability of data and material Not applicable.

Code availability Not applicable.

\section{Declarations}

Conflict of interest All authors declare that they have no conflict of interest.

\section{References}

Anastasiades, P. S., \& Kotsidis, K. (2013). The Challenges of Web 2.0 for Education in Greece. International Journal of Web-Based Learning and Teaching Technologies, 8(4), 19-33. https://doi.org/10. 4018/ijwltt.2013100102

Anastasiades, P. (2014). Research in e-learning in Greek Primary, Secondary and Higher Education: Review and perspectives (in GreeK). Open Education - The Journal for Open and Distance Education and Educational Technology, 10(1), 5-32. https://doi.org/10.12681/jode.9809

Anderson, T. (2003b). Modes of interaction in distance education: Recent developments and research questions. In M. G. Moore \& W. G. Anderson (Eds.), Handbook of distance education (pp. 129144). Lawrence Erlbaum Associates.

Anderson, T., \& Garrison, R. (1998). Learning in a networked world: New roles and responsibilties. In C. C. Gibson (Ed.), Distance Learners in Higher Education: Institutional responses for quality outcomes (pp. 97-112). Atwood Publishing.

Anderson, T. (2003a). Getting the mix right again: An updated and theoretical rationale for interaction. The International Review of Research in Open and Distributed Learning, 4(2). https://doi.org/10. 19173/irrodl.v4i2.149

Appana, S. (2008). A review of benefits and limitations of online learning in the context of the student, the instructor and the tenured faculty. International Journal on E-learning, 7(1), 5-22. Waynesville, NC USA: Association for the Advancement of Computing in Education (AACE). Retrieved November 20, 2021 from https://www.learntechlib.org/primary/p/22909/

Bartolic-Zlomislic, S., \& Bates, A. W. T. (1999). Investing in On-line Learning: Potential Benefits and Limitations. Canadian Journal of Communication, 24(3). https://doi.org/10.22230/cjc.1999v24n3a 1111

Bernard, R. M., Abrami, P. C., Borokhovski, E., Wade, C. A., Tamim, R. M., Surkes, M. A., \& Bethel, E. C. (2009). A meta-analysis of three types of interaction treatments in distance education. Review of Educational Research, 79(3), 1243-1289. https://doi.org/10.3102/0034654309333844

Bernard, R. M., Borokhovski, E., \& Tamim, R. (2019). The state of research on distance, online, and blended kearning: Meta-analyses and qualitative systematic reviews. In M. G. Moore \& W. C. Diehl (Eds.), Handbook of Distance Education (pp. 92-104). Routledge.

Blackmon, S. J., \& Major, C. (2012). Student experiences in online courses a qualitative research synthesis. Quarterly Review of Distance Education, 13(2).

Burnham, B. R., \& Walden, B. (1997). Interactions in distance education: A report from the other side. Proceedings of Adult Education Research Conference (pp. 49-54). Oklahoma State University.

Chakraborty, P., Mittal, P., Gupta, M. S., Yadav, S., \& Arora, A. (2021). Opinion of students on online education during the COVID-19 pandemic. Human Behavior and Emerging Technologies, 3, 357-365. https://doi.org/10.1002/hbe2.240

Drisko, J. W., \& Maschi, T. (2016). Content analysis. Oxford University Press.

Dumford, A. D., \& Miller, A. L. (2018). Online learning in higher education: Exploring advantages and disadvantages for engagement. Journal of Computing in Higher Education, 30(3), 452-465. https:// doi.org/10.1007/s12528-018-9179-z

Hillman, D. C. A., Willis, D. J., \& Gunawardena, C. N. (1994). Learner-interface interaction in distance education: An extension of contemporary models and strategies for practitioners. American Journal of Distance Education, 8(2), 30-42. https://doi.org/10.1080/08923649409526853

Howland, J. L., \& Moore, J. L. (2002). Student perceptions as distance learners in internet-based courses. Distance Education, 23(2), 183-195. https://doi.org/10.1080/0158791022000009196 
Joshi, O., Chapagain, B., Kharel, G., Poudyal, N. C., Murray, B. D., \& Mehmood, S. R. (2020). Benefits and challenges of online instruction in agriculture and natural resource education. Interactive Learning Environments, 1-12,. https://doi.org/10.1080/10494820.2020.1725896

Kang, H., \& Gyorke, A. S. (2008). Rethinking distance learning activities: A comparison of transactional distance theory and activity theory. Open Learning: The Journal of Open, Distance and e-Learning, 23(3), 203-214. https://doi.org/10.1080/02680510802420050

Kara, M. (2020). Transactional distance and learner outcomes in an online EFL context. Open Learning: The Journal of Open, Distance and e-Learning, 1-16,. https://doi.org/10.1080/02680513.2020.17174 54

Kassandrinou, A., Angelaki, C., \& Mavroidis, I. (2014). Transactional distance among Open University students: how does it affect the learning process. European Journal of Open, Distance and E-Learning, 17(1), 26-42. https://doi.org/10.2478/eurodl-2014-0002

Kebritchi, M., Lipschuetz, A., \& Santiague, L. (2017). Issues and challenges for teaching successful online courses in higher education. Journal of Educational Technology Systems, 46(1), 4-29. https://doi.org/ 10.1177/0047239516661713

Lincoln, Y., \& Guba, E. (1985). Naturalistic Inquiry. Sage.

Mishra, L., Gupta, T., \& Shree, A. (2020). Online teaching-learning in higher education during lockdown period of COVID-19 pandemic. International Journal of Educational Research Open, 1, 100012. https://doi.org/10.1016/j.ijedro.2020.100012

Moore, M. G. (1989). Three types of interaction. American Journal of Distance Education, 3(2), 1-7. https://doi.org/10.1080/08923648909526659

Moore, M. G. (1993). Theory of transactional distance. In D. Keegan (Ed.), Theoretical Principles of Distance Education (pp. 20-35). Routledge.

Moore, M. G., \& Kearsley, G. (2011). Distance education: A Systems View of Online Learning (3rd ed.). Wadsworth Cengage Learning.

Muilenburg, L. Y., \& Berge, Z. L. (2005). Student barriers to online learning: A factor analytic study. Distance Education, 26(1), 29-48. https://doi.org/10.1080/01587910500081269

Murphy, M. P. A. (2020). COVID-19 and emergency eLearning: Consequences of the securitization of higher education for post-pandemic pedagogy. Contemporary Security Policy, 41(3), 492-505. https:// doi.org/10.1080/13523260.2020.1761749

Naidu, S. (2020). It is the worst-and the best-of times. Distance Education, 41(4), 425-428. https://doi. org/10.1080/01587919.2020.1825929

Owusu-Boampong, A., \& Holmberg, C. (2015). Distance Education in European higher education: the potential. UNESCO, Institute for Lifelong Learning, International Council for Open and Distance Education. Retrieved November 20, 2021 from http://hdl.voced.edu.au/10707/396120

Patricia Aguilera-Hermida, A. (2020). College students' use and acceptance of emergency online learning due to COVID-19. International Journal of Educational Research Open, 1, 100011. https://doi.org/10. 1016/j.ijedro.2020.100011

Paul, R., Swart, W., Zhang, A. M., \& MacLeod, K. (2015). Revisiting Zhang's scale of transactional distance: Refinement and validation using structural equation modeling. Distance Education, 36(3), 364382. https://doi.org/10.1080/01587919.2015.1081741

Ronggui, H. (2016). RQDA: R-based Qualitative Data Analysis. R package version 0.2-8. http://rqda.rforge.r-project.org/.

Schreier, M. (2014). Qualitative Content Analysis. In U. Flick (Ed.), The SAGE Handbook of Qualitative Data Analysis (pp. 170-183). SAGE.

Teddlie, C., \& Tashakkori, A. (2009). Foundations of mixed methods research: Integrating quantitative and qualitative approaches in the social and behavioral sciences. Thousand Oaks, CA: Sage.

Publisher's Note Springer Nature remains neutral with regard to jurisdictional claims in published maps and institutional affiliations. 